\title{
A high-statistics study of the nucleon EM form factors, axial charge and quark momentum fraction
}

\section{B. Jäger; T.D. Rae†, S. Capitani, M. Della Morte, D. Djukanovic, G. von Hippel, B. Knippschild, H.B. Meyer, H. Wittig}

PRISMA Cluster of Excellence and Institut für Kernphysik, Becher-Weg 45, University of Mainz, D-55099 Mainz, Germany

Helmholtz Institute Mainz, University of Mainz, D-55099 Mainz, Germany

IFIC and CSIC, calle Catedratico Jose Beltran 2, 46980 Paterna, Spain

E-mail: jaegerakph.uni-mainz.de, thraeduni-mainz.de

We present updated results for the nucleon axial charge and electromagnetic (EM) form factors, which include a significant increase in statistics for all ensembles (up to 4000 measurements), as well as the addition of ensembles with pion masses down to $m_{\pi} \sim 195 \mathrm{MeV}$. We also present results for the average quark momentum fraction. The new data allows us to perform a thorough study of the systematic effects encountered in the lattice extraction. We concentrate on systematic effects due to excited-state contaminations for each of the quantities, which we check using several different time separations between the operators at the source and sink through a comparison of plateau fits and the summed operator insertion method (which provides a mechanism to suppress the excited-state contamination). We confirm our earlier finding [1] that a reliable extraction of the axial charge must be based on a method which eliminates excited-state contaminations. Similar conclusions apply to our EM form factor calculations [2]. The measurements are calculated using the CLS ensembles with non-perturbatively O(a) improved Wilson fermions in $N_{f}=2$ QCD.

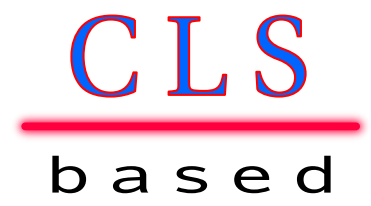

31st International Symposium on Lattice Field Theory LATTICE 2013

July 29 - August 3, 2013

Mainz, Germany

\footnotetext{
* Speaker.

$\dagger$ Speaker.
} 


\begin{tabular}{cccccccc}
\hline$\beta$ & $a[\mathrm{fm}]$ & lattice & $L[\mathrm{fm}]$ & $m_{\pi}[\mathrm{MeV}]$ & $m_{\pi} L$ & Label & $\#$ meas. \\
\hline 5.20 & 0.079 & $64 \times 32^{3}$ & 2.5 & 473 & 6.0 & A3 & 2128 \\
5.20 & 0.079 & $64 \times 32^{3}$ & 2.5 & 363 & 4.7 & A4 & 3200 \\
5.20 & 0.079 & $64 \times 32^{3}$ & 2.5 & 312 & 4.0 & A5 & 4000 \\
5.20 & 0.079 & $96 \times 48^{3}$ & 3.8 & 262 & 5.0 & B6 & 2544 \\
\hline 5.30 & 0.063 & $64 \times 32^{3}$ & 2.0 & 451 & 4.7 & E5 & 4000 \\
5.30 & 0.063 & $96 \times 48^{3}$ & 3.0 & 324 & 5.0 & F6 & 3600 \\
5.30 & 0.063 & $96 \times 48^{3}$ & 3.0 & 277 & 4.2 & F7 & 3000 \\
5.30 & 0.063 & $128 \times 64^{3}$ & 4.0 & 195 & 4.0 & G8 & 4176 \\
\hline 5.50 & 0.050 & $96 \times 48^{3}$ & 2.4 & 536 & 6.5 & N4 & 600 \\
5.50 & 0.050 & $96 \times 48^{3}$ & 2.4 & 430 & 5.2 & N5 & 1908 \\
5.50 & 0.050 & $96 \times 48^{3}$ & 2.4 & 340 & 4.0 & N6 & 3784 \\
5.50 & 0.050 & $128 \times 64^{3}$ & 3.2 & 270 & 4.4 & O7 & 1960 \\
\hline
\end{tabular}

Table 1: Details of the lattice ensembles used in this study, showing $\beta$-values, lattice spacing $a$ (determined in [21]), lattice extent $L$ (where $T=2 L$ ), pion mass $m_{\pi}$ and the total number of measurements.

\section{Introduction}

The axial charge of the nucleon is very well determined from experiment, $g_{A}=1.2701(25)$ [3], and provides a benchmark for Lattice QCD (LQCD) calculations, since it is constructed as a simple matrix element from a local operator with quark bilinears, involves no momentum in the initial and final states, and is an isovector quantity that has no quark-disconnected diagrams. However, the results from lattice calculations are typically $\sim 10 \%$ below the experimental value [4]-[20]. It is therefore important to ensure that systematic effects are under sufficient control. We have previously argued in [1] that this discrepancy can be explained by carefully accounting for excited states, for which we use the summation method (described in section 3). Similar methods have been used in [22]. This proceedings contribution provides an update to the results for our $g_{A}$ calculation [1], and to our nucleon electromagnetic (EM) form factors results [2, 23, 24]. The EM form factors are crucial observables in hadronic physics and provide details of the distribution of charge and magnetisation in the nucleon, for which a similar discrepancy between the lattice and experiment is seen as for $g_{A}[4,5],[12]-[20],[22,25]$. In addition to the quantities previously calculated by our group, we present first results for the quark momentum fraction $\langle x\rangle$ of the nucleon, which may also be considered a benchmark quantity and tends to be overestimated in lattice calculations [16, $18,20,22]$, [26]-[30]. Our simulations use non-perturbatively $\mathscr{O}(a)$ improved Wilson fermions in $N_{f}=2$ QCD, generated as part of the CLS effort. Table 1 provides details of the lattice ensembles.

The matrix element of a nucleon interacting with the axial current, $A_{\mu}=\bar{\psi}(x) \gamma_{5} \gamma^{\mu} \psi(x)$, may be decomposed into the axial and pseudoscalar form factors $G_{A}$ and $G_{P}$ :

$$
\left\langle N\left(p^{\prime}, s^{\prime}\right)\left|A_{\mu}\right| N(p, s)\right\rangle=\bar{u}\left(p^{\prime}, s^{\prime}\right)\left[\gamma_{\mu} \gamma_{5} G_{A}\left(Q^{2}\right)+\gamma_{5} \frac{q_{\mu}}{2 m_{N}} G_{P}\left(Q^{2}\right)\right] u(p, s),
$$

whereas for the electromagnetic current, $V^{\mu}=\bar{\psi}(x) \gamma^{\mu} \psi(x)$, the matrix element may be parame- 
terised by the Dirac and Pauli form factors $F_{1}$ and $F_{2}$ :

$$
\left\langle N\left(p^{\prime}, s^{\prime}\right)\left|V_{\mu}\right| N(p, s)\right\rangle=\bar{u}\left(p^{\prime}, s^{\prime}\right)\left[\gamma_{\mu} F_{1}\left(Q^{2}\right)+i \frac{\sigma_{\mu v} q_{v}}{2 m_{N}} F_{2}\left(Q^{2}\right)\right] u(p, s),
$$

where $u(p, s)$ is a Dirac spinor with spin $s$, and momentum $p, \gamma_{\mu}$ is a Dirac matrix, $\sigma_{\mu v}=\frac{1}{2 i}\left[\gamma_{\mu}, \gamma_{v}\right]$, and $Q^{2}=-\left(E_{p^{\prime}}-E_{p}\right)^{2}+\vec{q}^{2}$ where $\vec{q}=\vec{p}^{\prime}-\vec{p}$. The Pauli and Dirac form factors are related to the Sachs form factors $G_{E}$ and $G_{M}$,

$$
G_{E}\left(Q^{2}\right)=F_{1}\left(Q^{2}\right)-\frac{Q^{2}}{4 m_{N}^{2}} F_{2}\left(Q^{2}\right), \quad G_{M}\left(Q^{2}\right)=F_{1}\left(Q^{2}\right)+F_{2}\left(Q^{2}\right)
$$

that are measured in scattering experiments via the differential cross section described by the Rosenbluth formula. The form factors may be Taylor expanded in the momentum transfer $Q^{2}$,

$$
G_{X}\left(Q^{2}\right)=G_{X}(0)\left(1-\frac{1}{6}\left\langle r_{X}^{2}\right\rangle Q^{2}+\mathscr{O}\left(Q^{4}\right)\right),
$$

from which the charge radii of the nucleon may be determined:

$$
\left\langle r_{X}^{2}\right\rangle=-\left.\frac{6}{G_{X}\left(Q^{2}\right)} \frac{\partial G_{X}\left(Q^{2}\right)}{\partial Q^{2}}\right|_{Q=0},
$$

where $X=E, M$. Note that $G_{A}(0)=g_{A}$ and for the conserved current, $G_{E}(0)=1$ and $G_{M}(0)=\mu$, where $\mu$ measures the magnetic moment in nuclear magneton units $e /\left(2 m_{N}\right)$.

The hadronic matrix element containing a single derivative can be related to the generalised form factors $A_{20}, B_{20}$ and $C_{20}$ through

$$
\begin{aligned}
\left\langle N\left(p^{\prime}, s^{\prime}\right)\left|\gamma_{\{\mu} \stackrel{\leftrightarrow}{D}_{v\}}\right| N(p, s)\right\rangle=\bar{u}\left(p^{\prime}, s^{\prime}\right) & \left(\gamma_{\{\mu} Q_{v\}} A_{20}\left(Q^{2}\right)\right. \\
+ & \left.i \frac{\sigma_{\{\mu \alpha} Q_{\alpha} p_{v\}}}{2 m} B_{20}\left(q^{2}\right)+\frac{1}{m} p_{\{\mu} p_{\nu\}} C_{20}\left(Q^{2}\right)\right) u(p, s),
\end{aligned}
$$

where $\stackrel{\leftrightarrow}{D}_{v}=\vec{D}_{v}-\overleftarrow{D}_{v}$, and $A_{20}(0) \equiv\langle x\rangle$ is the average quark momentum fraction

\section{Lattice formulation}

The calculation of the form factors requires a ratio of correlation functions, for which we use

$$
R_{\gamma_{\mu}}\left(\vec{q}, t, t_{s}\right)=\frac{C_{3, \gamma_{\mu}}\left(\vec{q}, t, t_{s}\right)}{C_{2}\left(\overrightarrow{0}, t_{s}\right)} \sqrt{\frac{C_{2}\left(\vec{q}, t_{s}-t\right) C_{2}(\overrightarrow{0}, t) C_{2}\left(\overrightarrow{0}, t_{s}\right)}{C_{2}\left(\overrightarrow{0}, t_{s}-t\right) C_{2}(\vec{q}, t) C_{2}\left(\vec{q}, t_{s}\right)}},
$$

where $\vec{p}^{\prime}=0$. This ratio was found to be the most effective ratio of several studied in [31]. In the case $\vec{p}=\vec{q}=0$, which is relevant for $g_{A}$ and $\langle x\rangle$, eq. 2.1, simplifies as the square root factor equals 1. The two- and three-point functions $C_{2}(\vec{p}, t)$ and $C_{3, \gamma_{\mu}}\left(\vec{q}, t, t_{s}\right)$ are given by (fig. 1),

$$
\begin{aligned}
C_{2}(\vec{p}, t) & =\sum_{\vec{x}}\left\langle\Gamma_{\alpha^{\prime} \alpha} J_{\alpha}(x) \bar{J}_{\alpha^{\prime}}(0)\right\rangle e^{-i \vec{p} \cdot \vec{x}}, \\
C_{3, \gamma_{\mu}}\left(\vec{q}, t, t_{s}\right) & =\sum_{\vec{x}, \vec{y}}\left\langle\Gamma_{\alpha^{\prime} \alpha} J_{\alpha}\left(\vec{x}, t_{s}\right) \mathscr{O}_{\gamma_{\mu}}(\vec{y}, t) \bar{J}_{\alpha^{\prime}}(0)\right\rangle e^{-i \vec{q} \cdot \vec{y}},
\end{aligned}
$$



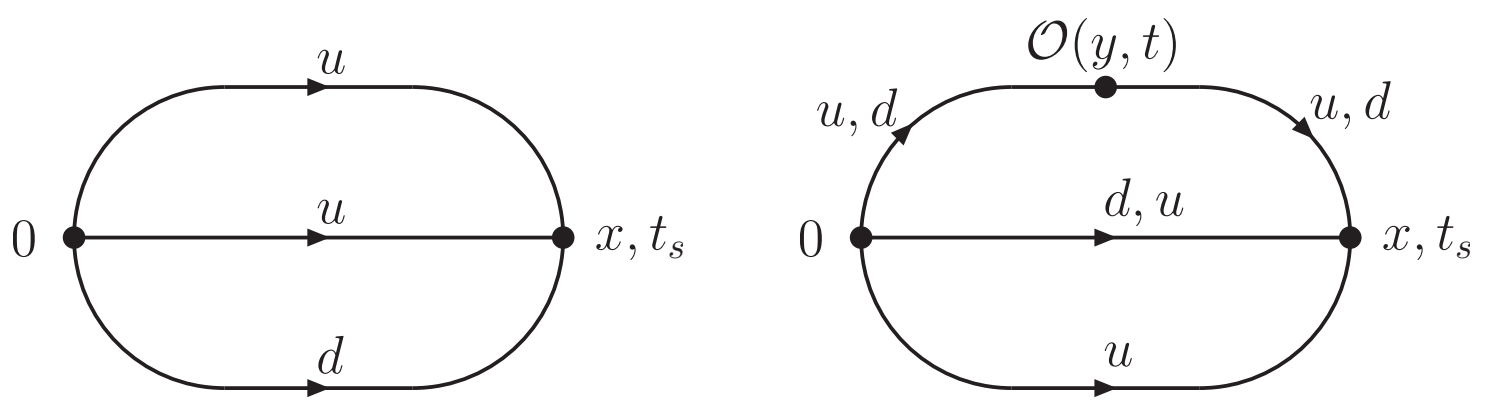

Figure 1: Schematic diagrams for the two- and three-point functions, left and right panels respectively.

where $J_{\alpha}(x)$ is a suitably chosen interpolating operator with the correct quantum numbers to create a nucleon, and $\Gamma_{\alpha \alpha^{\prime}}$ is a projection matrix used to give the interpolating fields the correct parity. We chose to polarise the nucleon in the $z$-direction, $\Gamma=\frac{1}{2}\left(1+\gamma_{0}\right)\left(1+i \gamma_{5} \gamma_{3}\right)$. We consider both local and conserved vector currents, where the latter is defined as

$$
\mathscr{O}_{\mu}^{\mathrm{con}}(x)=\frac{1}{2}\left(\bar{\psi}(x+a \hat{\mu})\left(1+\gamma_{\mu}\right) U_{\mu}^{\dagger}(x) \psi(x)-\bar{\psi}(x)\left(1-\gamma_{\mu}\right) U_{\mu}(x) \psi(x+a \hat{\mu})\right)
$$

where $\psi=u, d$. In principle, we are able to determine the EM form factors and $\langle x\rangle$ for the proton and for the neutron, depending on the linear combination of contributions from the quark correlation functions. However, here we focus on the iso-vector combination for which the quarkdisconnected diagrams cancel. To improve the overlap of the interpolating operators with the nucleon, we use Gaussian smearing [32], supplemented by APE smeared links [33], at both source and sink.

The calculation of the three-point function involves the insertion of an operator at time $t$; to do this we use the 'fixed sink method', which fixes the final and initial states whilst allowing both the operator and momentum transfer to be chosen without the need for additional inversions [34]. Our specific choice of kinematics $\vec{p}^{\prime}=0$, and thus $\vec{p}=-\vec{q}$, allow us to extract all vector form factors $G_{E}, G_{M},\left(F_{1}, F_{2}\right)$ as well as both the axial charge $g_{A}$ and $\langle x\rangle$ (when $\vec{q} \rightarrow 0$ ) from eq. (2.1) at large time arguments,

$$
\begin{gathered}
R_{\gamma_{5} \gamma_{3}}\left(\vec{q}=0, t, t_{s}\right)=g_{A}, \\
R_{\mathscr{O}_{\langle x\rangle}\left(\vec{q}=0, t, t_{s}\right)=m_{N}\langle x\rangle^{\text {bare }},} \\
R_{\gamma_{0}}\left(\vec{q}, t, t_{s}\right)=\sqrt{\frac{M+E}{2 E}} G_{E}\left(Q^{2}\right), \\
R_{\gamma_{i}}\left(\vec{q}, t, t_{s}\right)=\varepsilon_{i j} p_{j} \sqrt{\frac{1}{2 E(E+M)}} G_{M}\left(Q^{2}\right), \quad i=1,2 .
\end{gathered}
$$

\section{Systematics of extraction}

In order to have an unbiased determination of the quantities of interest, the correlation functions must have reached their asymptotic behaviour. If the asymptotic behaviour has not been reached simple plateau fits will show a systematic trend that is dependent on the source-sink separations 
$t_{s}$ (fig. 2). A priori it is not possible to know the appropriate source-sink separation for a given quantity, which also depends on the projection properties of the nucleon interpolating operators.

For all our ensembles we use four separate source-sink separations $(\sim 0.6-1.2 \mathrm{fm})$, and even at the largest $t_{s} \sim 1.1 \mathrm{fm}$ it is not clear from a plateau fit that the contaminations from excited states are sufficiently suppressed for an unbiased determination of the quantities. Therefore, for the 'N6' ensemble we extended the number of source-sink separations from four to six, increasing the source-sink separation range up to $t_{s} / a=28(1.4 \mathrm{fm})$, see fig. 2 . Even for this extended range, it is difficult to determine that the data has reached the asymptotic behaviour, before the signal is lost for the largest $t_{s} / a=28$, as can be seen from the systematic trend in the data sets. It is therefore important to take the excited states into account to have a good handle on possible systematic errors. The excited-state contributions to the ratio may be factorised from the ground state contributions, so that

$$
R\left(\vec{q}, t, t_{s}\right)=R^{0}\left(\vec{q}, t, t_{s}\right)\left(1+\mathscr{O}\left(e^{-\Delta t}\right)+\mathscr{O}\left(e^{-\Delta^{\prime}\left(t_{s}-t\right)}\right)\right)
$$

where $\Delta$ and $\Delta^{\prime}$ are the energy gaps of the initial and final nucleons respectively. The method of summed operator insertions [35],

$$
S\left(t_{s}\right)=\sum_{t=0}^{t_{s}} R\left(\vec{q}, t, t_{s}\right) \rightarrow c\left(\Delta, \Delta^{\prime}\right)+t_{s}\left(G_{E, M}+\mathscr{O}\left(e^{-\Delta t_{s}}\right)+\mathscr{O}\left(e^{-\Delta^{\prime} t_{s}}\right)\right),
$$

allows the form factors to be extracted from the slope after computing $S\left(t_{s}\right)$ for several $t_{s}$. The results for the summation method are overlaid in yellow in figs. 2 and 3 and tend to agree or are minimally overlapping with the data for $t_{s} / a=25$, corresponding to $t_{s}=1.25 \mathrm{fm}$, therefore indicating that the asymptotic behaviour has not yet been reached. A common method is to fit the largest $t_{s}$ data with a plateau. However it is difficult, as mentioned, to know a priori if the source-sink separation is 'large enough', whereas the summation method has the advantage that the excited states are parametrically reduced and there is no need to fit a plateau to what can sometimes be very noisy data, especially for large $t_{s}$ and large $Q^{2}$. Also, the summation method only requires linear fits, whereas any extension of plateau fits to include excited states would imply non-linear (and therefore possibly unstable) fits.

Another important consideration besides the source-sink separations, is the question of: what is 'enough' statistics in order to satisfactorily resolve the desired quantity? To check this, on the 'F7' ensemble $\left(m_{\pi}=277 \mathrm{MeV}\right)$, we show $g_{A}$ and $G_{E}\left(Q^{2}=\left(\frac{2 \pi}{L a}\right)^{2}\right)$ with both 1000 and 3000 measurements (fig. 3). The results for 1000 measurements suggest that the largest $t_{s}$ for the plateau method is 'large enough' as the $t_{s}$ dependence appears to have saturated. However, when the statistics are increased to 3000 measurements we clearly see that this is not the case, as indicated by the reduced overlap between the summation method and the individual $t_{s}$ data sets in both quantities.

\section{Electromagnetic form factor $Q^{2}$ dependence}

For the discussion of the electromagnetic form factors we concentrate on the conserved current as this removes the requirement of any renormalisation for the lattice operators; however, we note 

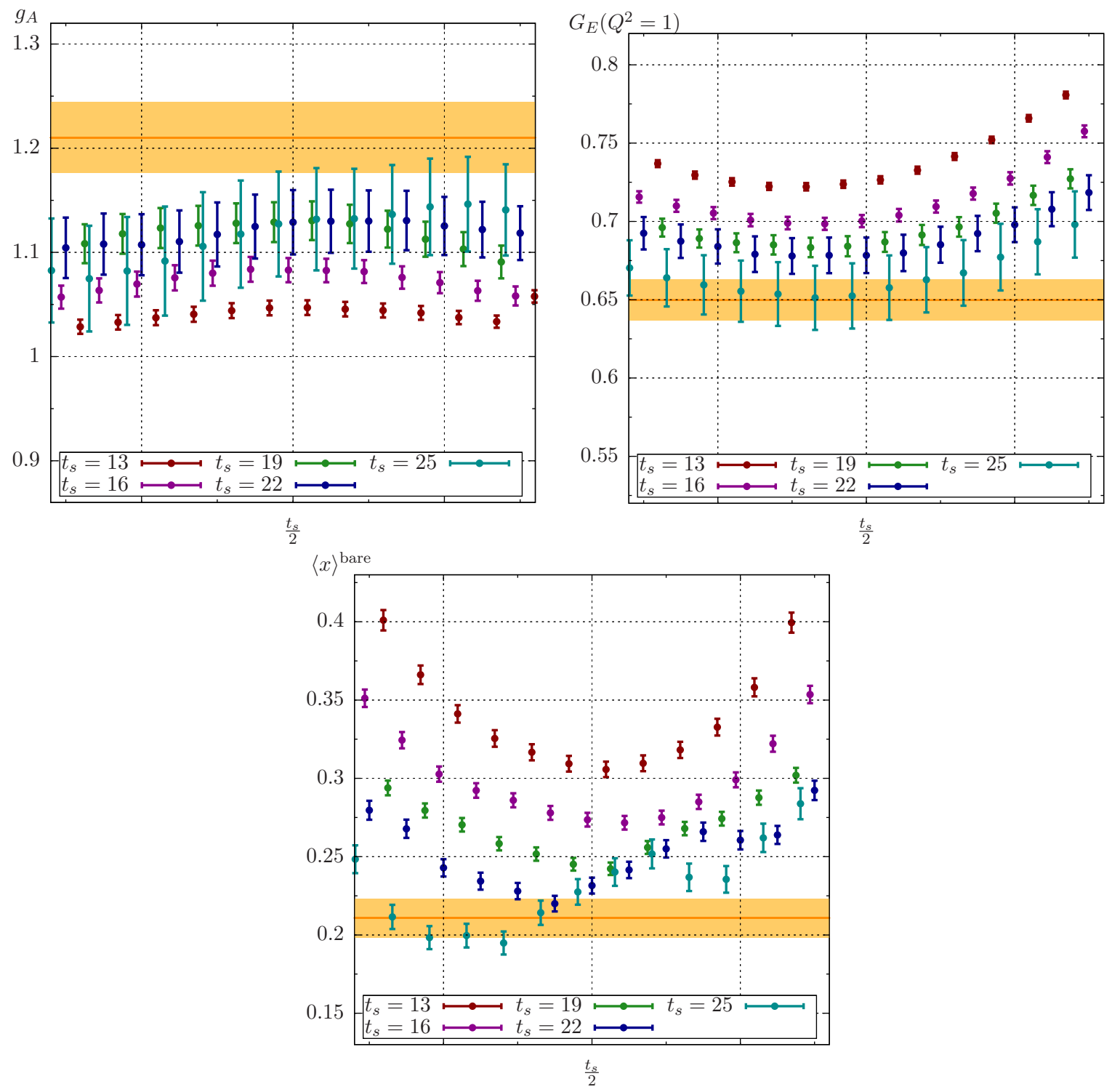

Figure 2: $g_{A}, G_{E}\left(Q^{2}=1\left(\frac{2 \pi}{L a}\right)^{2}\right)$ and $\langle x\rangle^{\text {bare }}$ for different source-sink separations, $t_{s}$, indicated by the legend. The summation method result is given by the yellow band. All panels are shown for our 'N6' ensemble $\left(m_{\pi}=340 \mathrm{MeV}\right)$. Due to the large statistical noise the largest $t_{s} / a=28$ data has been removed.

that a comparison between the local and conserved current provides a check of the renormalisation factor, which we find to be in agreement with other work (such as [36]).

To model the $Q^{2}$ dependence of the form factors, shown for $G_{E}$ in fig. 4, we use a dipole ansatz

$$
G_{E, M}\left(Q^{2}\right)=G_{E, M}(0) /\left(1+Q^{2} / M_{E, M}^{2}\right)^{2},
$$

shown in fig. 5 (for the 'O7' ensemble, $m_{\pi}=270 \mathrm{MeV}$ ) for $G_{E}$ and $G_{M}$ alongside the Kelly parameterisation [37] of the experimental data. It should be noted that in order for the lattice data and experimental parameterisation to be fully compatible a chiral extrapolation of the lattice data 

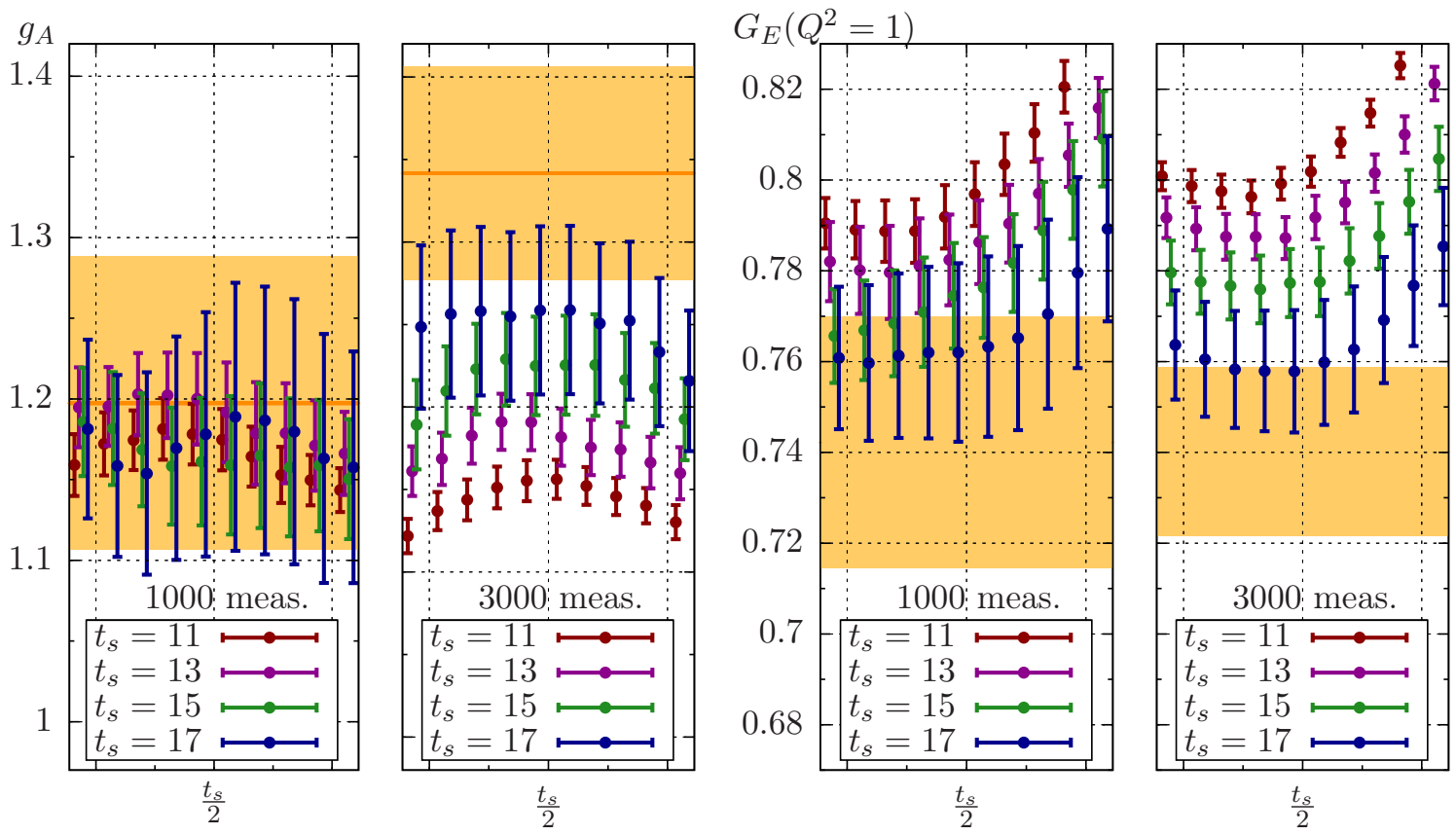

Figure 3: Left panels: $g_{A}$. Right panels: $G_{E}\left(Q^{2}=1\left(\frac{2 \pi}{L a}\right)^{2}\right)$. For each the left sub panel shows the quantity for 1000 measurements and the right sub-panel for 3000 measurements. Shown for the 'F7' ensemble $\left(m_{\pi}=277 \mathrm{MeV}\right)$.

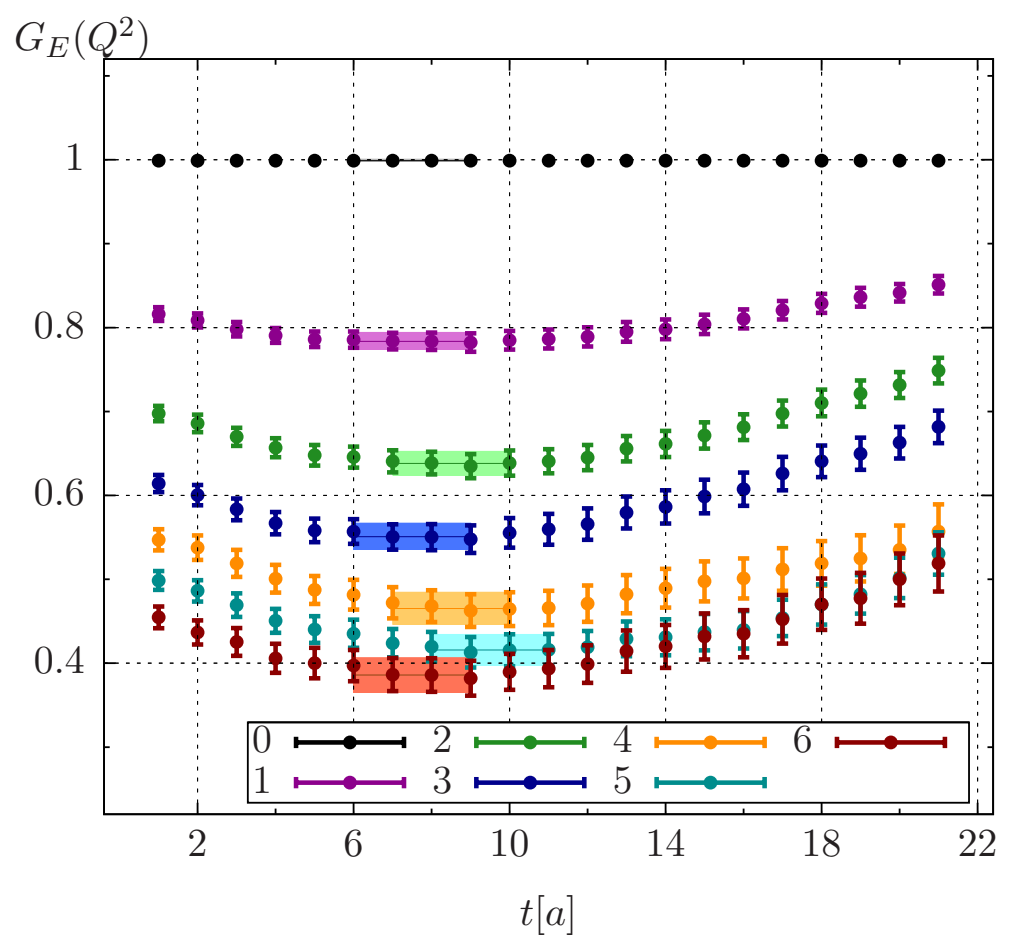

Figure 4: $G_{E}$ for each $Q^{2}=n\left(\frac{2 \pi}{L a}\right)^{2}$ where the legend gives the value of $n$. Shown for the largest $t_{s} \sim 1.1 \mathrm{fm}$, for the 'O7' ensemble $m_{\pi}=270 \mathrm{MeV}$. 
is required. In the case of $G_{E}$ we see a better agreement with the Kelly parameterisation [37] for the summation method than for a plateau fit at the largest $t_{s} \sim 1.1 \mathrm{fm}$, especially at large $Q^{2}$. However, for the case of $G_{M}$ it is harder to disentangle the plateau and summation methods, and further study is required to determine whether or not this is indicative that the asymptotic behaviour has been reached, or if still more statistics are required. Due to the extra momentum factor required in the extraction of $G_{M}$ (see eq. 2.8), its statistical accuracy is worse than that of $G_{E}$. The charge radii can be extracted from the dipole mass. However, due to the absence of a measured point at $G_{M}\left(Q^{2}=0\right)$, the determination of the radius, which is effectively the slope of the form factor at $Q^{2}=0$, is less constrained for $\left\langle r_{M}^{2}\right\rangle$ than $\left\langle r_{E}^{2}\right\rangle$, as can also be seen in fig. 5 .

We may obtain the magnetic moment $\mu$ from $G_{M}\left(Q^{2}=0\right)$ and also from the ratio

$$
M\left(Q^{2}\right)=\frac{G_{M}\left(Q^{2}\right)}{G_{E}\left(Q^{2}\right)}, \quad \text { where } \quad \mu=M(0)=1+\kappa
$$

shown in fig. 5. The effect of this ratio is to cancel the $Q^{2}$ behaviour, indicating that the form factors $G_{E}$ and $G_{M}$ have a very similar shape and hence their radii are quite similar. We can therefore extract $\mu$ from a constant fit to the data, which is compatible within errors to $G_{M}\left(Q^{2}=0\right)$ for both the summation and plateau methods.

\section{Chiral dependence of the EM form factors and axial charge}

The ensembles listed in table 1 cover a range of pion masses, from 195 to $650 \mathrm{MeV}$, enabling us to both perform extrapolations in the pion mass to the physical points and to check finite-volume and discretisation effects for all quantities. All of the chiral dependence plots figs. 6 and 7 show the data for different lattice spacings in different colours, given in the legend. The experimental value is shown by a black cross at the physical point (yellow vertical line).

The individual data points in fig. 6 exhibit only a mild $m_{\pi}$ dependence, and so a linear fit of the form

$$
A+B m_{\pi}^{2}
$$

may be appropriate to model the $g_{A}$ data. To check the stability of the chiral extrapolation to the entire pion mass range, we have applied a cut at $m_{\pi}=360 \mathrm{MeV}$, for which we see that the two extrapolations agree very well within statistical precision. In addition we see no obvious finite-size or discretisation effects; the latter has been checked with the addition of an $a^{2}$ term to the fits. If the excited states are taken into account via the summation method, we obtain a value for $g_{A}$ that is compatible with the experimental result. By contrast, using the plateau method with a source-sink separation of $\sim 1.1 \mathrm{fm}$ yields discrepancy with the experimental result [3], which is larger than $1 \sigma$, regardless of the pion mass range used for the extrapolation. Therefore, the summation method provides strong evidence that excited states need to be sufficiently accounted for to reach agreement with experimental values

The chiral dependence for $\left\langle r_{E}^{2}\right\rangle,\left\langle r_{M}^{2}\right\rangle$ and $\kappa$ are shown in fig. 7. With the exception of $\left\langle r_{M}^{2}\right\rangle$, the comparison of the plateau and summation method indicates that, as for $g_{A}$, it is necessary to account 

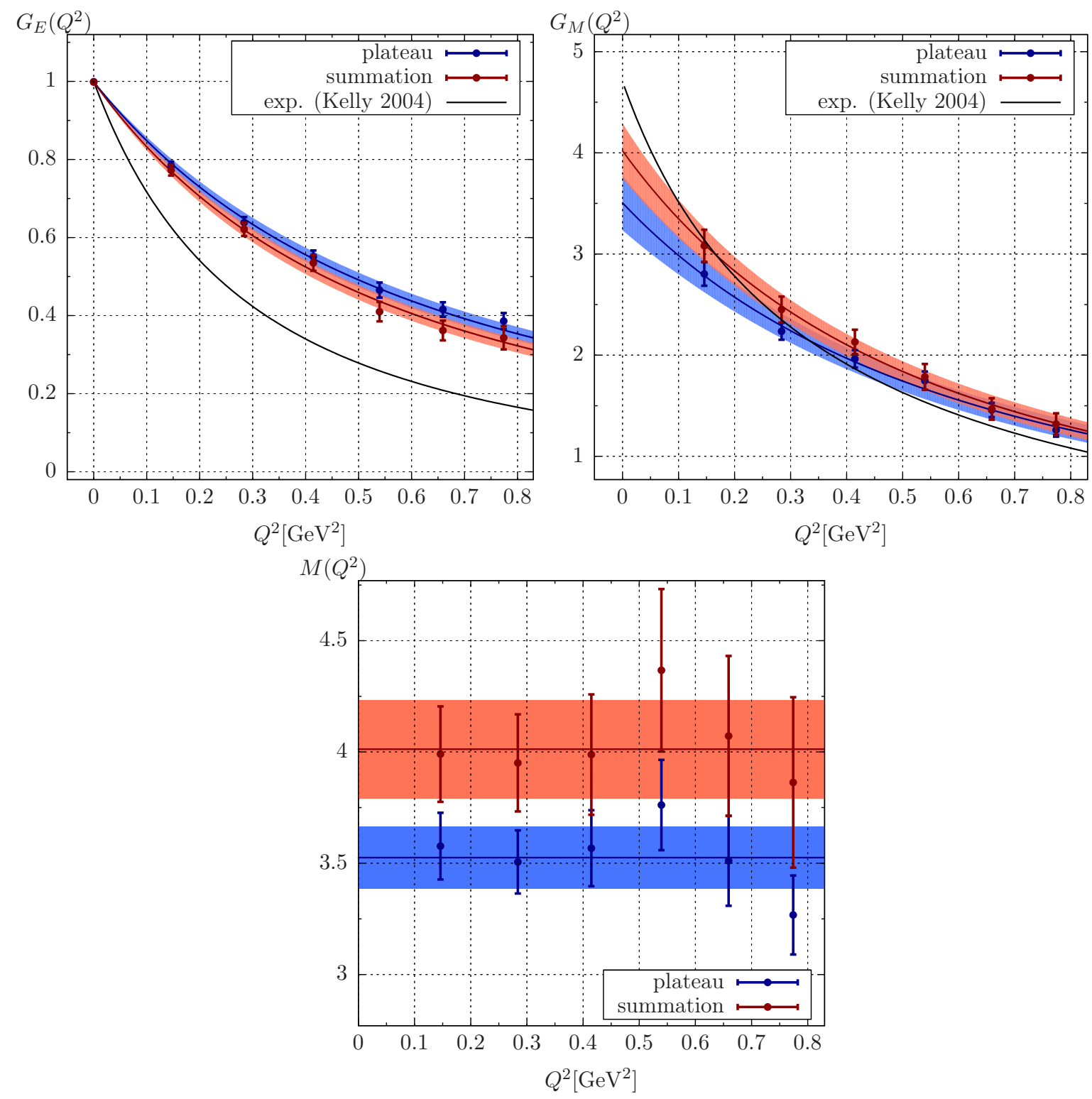

Figure 5: The top left and right panels show the $Q^{2}$ dependence of $G_{E}$ and $G_{M}$ respectively, which may be compared to the Kelly parameterisation [37] of the experimental data. The bottom panel shows $M\left(Q^{2}\right)$. The lattice data is for the 'O7' ensemble $m_{\pi}=270 \mathrm{MeV}$.

for excited states. For $\left\langle r_{M}^{2}\right\rangle$, we see that any extrapolations to the physical point will be strongly dependent upon the most chiral point and we note that larger statistical errors and fluctuations within the $\left\langle r_{M}^{2}\right\rangle$ data are largely due to the absence of a point equivalent to $G_{E}\left(Q^{2}=0\right)=1$, which helps to constrain both the $Q^{2}$ behaviour and the determination of the charge radius. As for $g_{A}$, the EM form factor data also shows no obvious finite volume or discretisation effects. We are currently exploring the effect of other fit forms on the results in figs. 6 and 7, including ansätze based on HBChPT, so as to have a comprehensive picture of the systematic effects. This will be commented upon in a forthcoming paper [38]. 

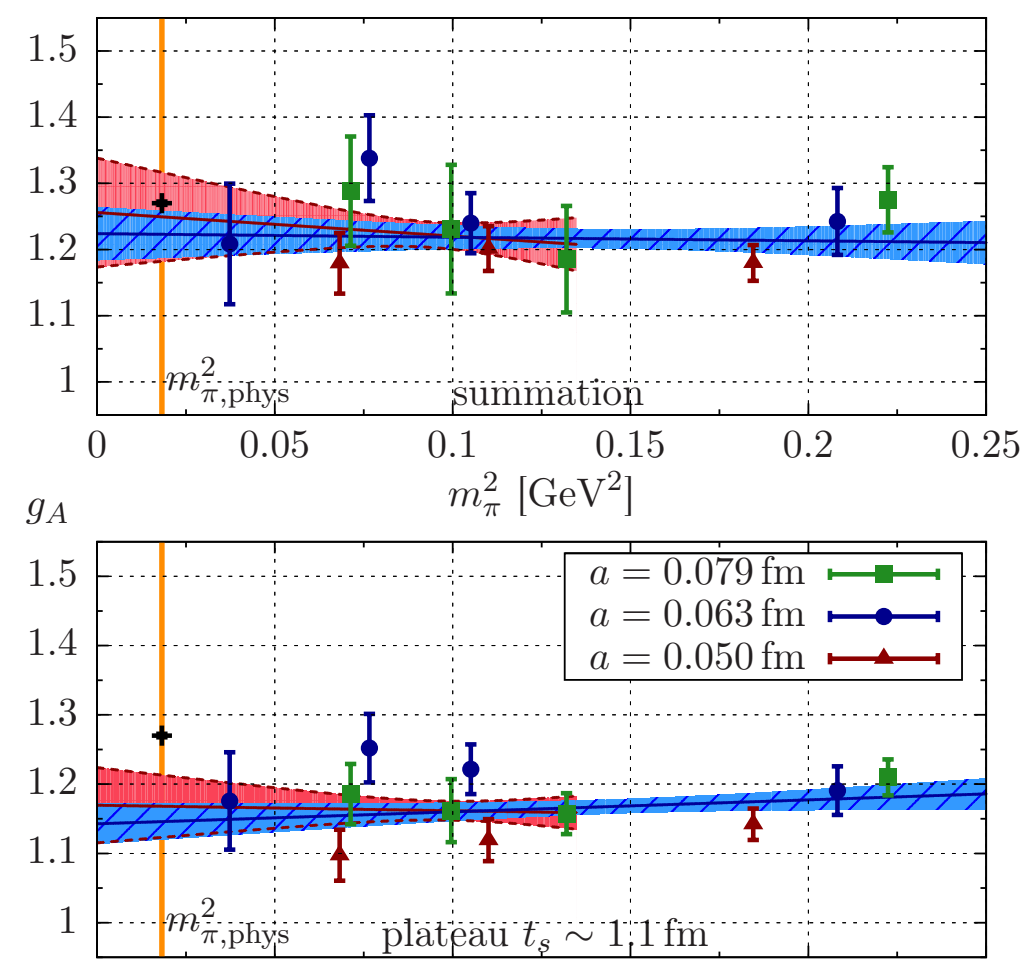

Figure 6: Chiral extrapolation of $g_{A}$ to the physical point (vertical yellow line). The black cross shows the experimental result [3] and the different symbols indicate the lattice spacing (see legend). The blue band shows a linear fit to the entire range, whereas the red band shows a linear fit with a mass cut at $m_{\pi}=360 \mathrm{MeV}$.

\section{Conclusions and outlook}

We have presented preliminary results for the nucleon's axial charge, vector form factors and quark momentum fraction with a focus on systematic errors due to excited state contaminations. Chiral extrapolations for the axial charge show that the use of the summed insertions method effectively accounts for contaminations from excited states, leading to good agreement with experiment. However, whilst we note that we are still analysing the chiral behaviour for this and the electromagnetic form factors, our data indicates that with sufficient statistics excited-state effects can be resolved and we present evidence that the summation method is an important tool to control the associated systematic errors. Further to this, we see no obvious finite size or discretisation effects in our data for all quantities.

For the average quark momentum fraction $\langle x\rangle$, which we have, so far, only evaluated at the bare ratio level, we see a similar effect as is seen for the EM form factors and axial charge, and conclude that excited-state effects appear to be equally important here, and reliably controlling them could help improve agreement with experiment as has proven to be the case for most of the other quantities discussed here. The average quark momentum fraction will be fully analysed including chiral fits, once a calculation of the required renormalisation constants using a non-perturbative scheme is completed. 

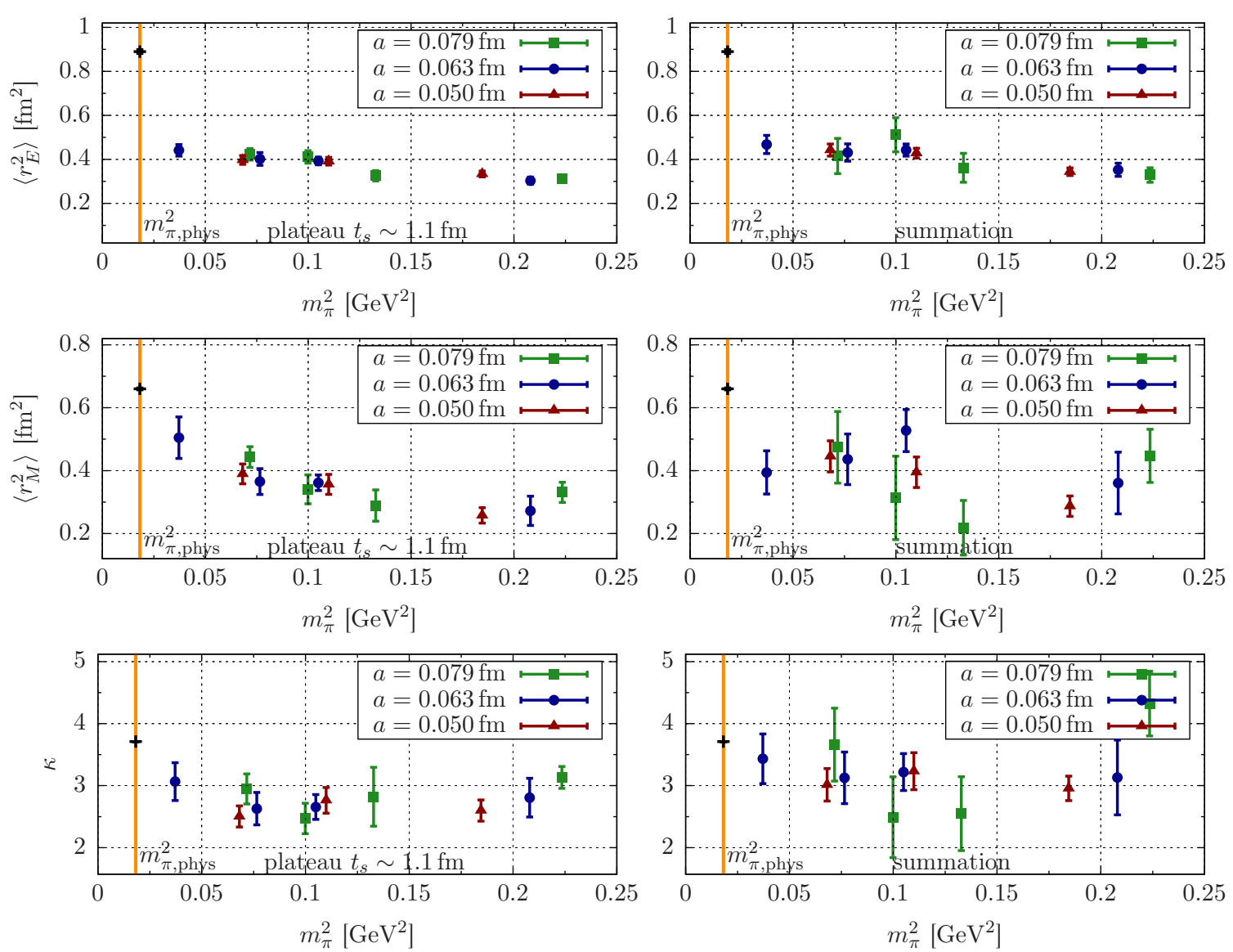

Figure 7: Chiral dependence of $\left\langle r_{E}^{2}\right\rangle,\left\langle r_{M}^{2}\right\rangle$ and $\kappa$. The vertical yellow line shows the physical point and the black cross the experimental result [3]. The different symbols indicate the lattice spacing (see legend).

The axial charge and form factors analysis is in the process of being finalised and will appear in an upcoming publication [38], therefore all results in this proceedings should, at the moment, be considered preliminary.

\section{Acknowledgments}

Our calculations were performed on the "Wilson" HPC Cluster at the Institute for Nuclear Physics, University of Mainz. We thank Christian Seiwerth for technical support. We are grateful for computer time allocated to project HMZ21 on the BG/Q "JUQUEEN" computer at NIC, Jülich. This work was granted access to the HPC resources of the Gauss Center for Supercomputing at Forschungzentrum Jülich, Germany, made available within the Distributed European Computing Initiative by the PRACE-2IP, receiving funding from the European Community's Seventh Framework Programme (FP7/2007-2013) under grant agreement RI-283493. This work was supported by the DFG via SFB 1044 and grant HA 4470/3-1. We are grateful to our colleagues within the CLS initiative for sharing ensembles. 


\section{References}

[1] S. Capitani et al., Phys. Rev. D86 074502 (2012), arXiv:1205.0180.

[2] S. Capitani et al., PoS LATTICE 2012177 (2012), arXiv:1211.1282.

[3] J. Beringer et al., (Particle Data Group), Phys. Rev. D86 010001 (2012).

[4] C. Alexandrou, PoS LATTICE 2010001 (2010), arXiv:1011.3660.

[5] D. B. Renner, PoS LATTICE 2009018 (2009), arXiv:1002.0925.

[6] G. Colangelo et al., Eur. Phys. J. C71, 1695 (2011), arXiv:1011.4408.

[7] LHP Collaboration, D. Dolgov et al., Phys. Rev. D66, 034506 (2002), hep-lat/0201021.

[8] RBCK Collaboration, S. Ohta and K. Orginos, Nucl. Phys. Proc. Suppl. 140, 396 (2005), hep-lat/0411008.

[9] LHP Collaboration, R. G. Edwards et al., Phys. Rev. Lett. 96, 052001 (2006), hep-lat/0510062.

[10] A.A. Khan et al., Phys. Rev. D74, 094508 (2006), hep-lat/0603028.

[11] RBC/UKQCD Collaboration, T. Yamazaki et al., Phys. Rev. Lett. 100, 171602 (2008), arXiv:0801.4016.

[12] H.-W. Lin et al., Phys. Rev. D78, 014505 (2008), arXiv:0802.0863.

[13] T. Yamazaki et al., Phys. Rev. D79, 114505 (2009), arXiv:0904.2039.

[14] G. Engel et al., PoS LATTICE 2009, 135 (2009), arXiv:0910.4190.

[15] QCDSF/UKQCD Collaboration, M. Göckeler et al., PoS LATTICE 2009, 125 (2009), arXiv:0912.0167.

[16] LHP Collaboration, J. D. Bratt et al., Phys. Rev. D82, 094502 (2010), arXiv:1001.3620.

[17] ETM Collaboration, C. Alexandrou et al., Phys. Rev. D83, 045010 (2011), arXiv:1012.0857.

[18] QCDSF/UKQCD Collaboration, D. Pleiter et al., PoS LATTICE 2010, 153 (2010), arXiv:1101.2326.

[19] QCDSF/UKQCD Collaboration, M. Göckeler et al., PoS LATTICE 2010, 163 (2010), arXiv:1102.3407.

[20] S. Syritsyn. http://www.lattice2013.uni-mainz.de/presentations/Plenaries\%20Thursday/Syritsyn.pdf (2013).

[21] S. Capitani et al., PoS LATTICE 2011 (2011) 145, arXiv:1110.6365.

[22] J. R. Green et al., arXiv:1209.1687.

[23] S. Capitani et al., PoS LATTICE 2010 (2010) 147, arXiv:1011.1358.

[24] S. Capitani et al., PoS ConfinementX (2012) 321.

[25] S. Collins et al., Phys. Rev. D84 (2011) 074507, arXiv:1106.3580.

[26] S. N. Syritsyn et al., Phys. Rev. D81 (2010) 034507, arXiv:0907.4194.

[27] G. S. Bali et al., Phys. Rev. D86 (2012) 054504, arXiv:1207.1110.

[28] A. Sternbeck et al., PoS LATTICE 2011 (2011) 177, arXiv:1203.6579.

[29] C. Alexandrou et al., Phys. Rev. D83 (2011) 114513, arXiv:1104.1600. 
[30] Y. Aoki et al., Phys. Rev. D82 (2010) 014501, arXiv:1003.3387.

[31] C. Alexandrou et al., PoS LATTICE 2008 (2008) 139, arXiv:0811.0724.

[32] S. Güsken et al., Phys. Lett. B227, 266 (1989).

[33] M. Albanese et al. [APE Collaboration], Phys. Lett. B192 (1987) 163.

[34] G. Martinelli and C. T. Sachrajda, Nucl. Phys. B316 (1989) 355.

[35] L. Maiani et al., Nucl. Phys. B293, 420 (1987).

[36] M. Della Morte et al., JHEP 0507 (2005) 007, hep-lat/0505026.

[37] J. J. Kelly, Phys. Rev. C70 (2004) 068202.

[38] S. Capitani et al., In preparation

[39] M. Göckeler et al., [QCDSF Collaboration], Phys. Rev. D71 (2005) 034508, hep-lat/0303019.

[40] J. D. Bratt et al., [LHPC Collaboration], Phys. Rev. D82 (2010) 094502, [arXiv:1001.3620.

[41] T. Yamazaki et al., Phys. Rev. D79 (2009) 114505, arXiv:0904.2039.

[42] A. A. Khan et al., Phys. Rev. D74 (2006) 094508, hep-lat/0603028.

[43] C. Alexandrou et al., Phys. Rev. D83 (2011) 094502, arXiv:1102.2208.

[44] R. Horsley et al., arXiv:1302.2233.

[45] H. -W. Lin, PoS LATTICE 2012 (2012) 013, arXiv:1212.6849.

[46] H. -W. Lin, arXiv:1112.2435. 